\title{
Parallel Programming and Parallel Abstractions in Fortress
}

\author{
Guy L. Steele \\ Sun Microsystems Laboratories
}

\begin{abstract}
The Programming Language Research Group at Sun Microsystems Laboratories seeks to apply lessons learned from the Java (TM) Programming Language to the next generation of programming languages. The Java language supports platform-independent parallel programming with explicit multithreading and explicit locks. As part of the DARPA program for High Productivity Computing Systems, we are developing Fortress, a language intended to support large-scale scientific computation. One of the design principles is that parallelism be encouraged everywhere (for example, it is intentionally just a little bit harder to write a sequential loop than a parallel loop). Another is to have rich mechanisms for encapsulation and abstraction; the idea is to have a fairly complicated language for library writers that enables them to write libraries that present a relatively simple set of interfaces to the application programmer. We will discuss ideas for using a rich polymorphic type system to organize multithreading and data distribution on large parallel machines. The net result is similar in some ways to data distribution facilities in other languages such as HPF and Chapel, but more openended, because in Fortress the facilities are defined by user-replaceable libraries rather than wired into the compiler.
\end{abstract}

